\title{
Microscopy Method to Compare Cyst Nematode Infection of Different Plant Species
}

\author{
Rinu Kooliyottil1, Louise-Marie Dandurand1*, Byju N. Govindan², Guy R. Knudsen ${ }^{1}$ \\ ${ }^{1}$ Department of Plant Soil and Entomological Sciences, University of Idaho, Moscow, ID, USA \\ ${ }^{2}$ Biological Systems Engineering, Washington State University, Pullman, WA, USA \\ Email: "Imd@uidaho.edu
}

Received 27 April 2016; accepted 27 June 2016; published 30 June 2016

Copyright (C) 2016 by authors and Scientific Research Publishing Inc.

This work is licensed under the Creative Commons Attribution International License (CC BY).

http://creativecommons.org/licenses/by/4.0/

(c) (i) Open Access

\begin{abstract}
The cyst nematode, Globodera pallida, is an obligate, biotrophic pathogen of potato, causing up to $\mathbf{8 0} \%$ yield loss. In the present study, a non-destructive imaging technique was used to compare the development and behavior of G. pallida in its host Solanum tuberosum and in the non-host $S$. sisymbriifolium. We used microscopy-rhizosphere chambers coupled with the fluorescent stain PKH26, and compared this with destructively sampled acid fuchsin staining. No significant difference $(P \geq 0.90)$ in $G$. pallida numbers was found whether stained with PKH26 or acid fuchsin for either plant species indicating no toxic effect from the vital stain. PKH26 labelled J2s successfully located and penetrated roots of both $S$. tuberosum and $S$. sisymbriifolium. Two days after inoculation, PKH26 stained G. pallida was clearly observed migrating intercellularly through root tissues of both $S$. tuberosum and the non-host $S$. sisymbriifolium. Overall, more nematodes were observed in $S$. tuberosum than in $S$. sisymbriifolium roots. No live J2s were observed in $S$. sisymbriifolium roots stained with either acid fuchsin or PKH26 after 8 days. Understanding the time line of development of G. pallida in S. sisymbriifolium is important towards comprehensive understanding of plant defense responses.
\end{abstract}

\section{Keywords}

Cyst Nematode, Solanum sisymbriifolium, Non-Destructive Imaging

\section{Introduction}

Plant parasitic nematodes cause major crop losses worldwide. They attack roots and other plant parts, reducing

${ }^{*}$ Corresponding author.

How to cite this paper: Kooliyottil, R., Dandurand, L.-M., Govindan, B.N. and Knudsen, G.R. (2016) Microscopy Method to Compare Cyst Nematode Infection of Different Plant Species. Advances in Bioscience and Biotechnology, 7, 311-318.

http://dx.doi.org/10.4236/abb.2016.76029 
water and nutrient uptake, and sapping plant vigor. Cyst nematodes in the genera Globodera and Heterodera are especially difficult to control, because the eggs can persist in soil for many years inside the dead bodies of females, which are called cysts. The potato cyst nematodes (PCN) Globodera pallida (Stone) Behrens and Globodera rostochiensis (Wollenweber) Behrens are major threats to potato production worldwide. Globodera pallida was first detected in the United States in 2006 in Idaho, and is regulated by both USDA-APHIS and the Idaho State Department of Agriculture.

Phytopathogenic nematodes go through six developmental stages: egg, four juvenile stages (J1 - J4), and adult. With G. pallida, up to four life stages may be present in plant roots: J2 - J4 and adult, depending on host susceptibility. The second stage juvenile is the infective stage; it migrates within the root, where it subsequently molts and initiates a feeding site. Once a feeding site is established, the nematode continues to develop into the adult stage. Both males and females are present. Adult males exit the root, then fertilize the sedentary females. After eggs are formed, the female dies, and her body serves as a protective "cyst” at the root surface. Nematode development is arrested at some point in resistant or non-host plants.

One potential control measure for PCN is the use of so-called trap crops, which are plant species that permit infection but do not support nematode development and reproduction. Solanum sisymbriifolium Lam, a nontuber-bearing solanaceous plant, has been identified as a promising trap crop for the control of G. pallida [1]. Solanum sisymbriifolium stimulates egg hatching but does not support reproduction of G. pallida [1]. Lack of development of G. pallida in roots of S. sisymbriifolium was observed by Sasaki-Crawley et al. [2], but observations were made by destructive sampling and staining. Ouden [3] used polyethylene bags filled with thin layers of foamed agar to observe nematode behavior of Heterodera rostochiensis, Hoplolaimus uniformis and Pratylenchus pratensis in potatoes, carrots and wheat, respectively. Atkinson et al. [4] studied the size and shape of $G$. pallida and Meloidogyne incognita during development in transgenic tomato by using a morphometric analysis method.

Novel approaches are required to study in vivo dynamics of G. pallida infection processes in potato as opposed to trap crops, and also to investigate potential mechanisms of resistance in different potato cultivars.

For live observation and behavioral studies of plant parasitic nematodes, fluorescent compounds such as fluorescein diacetate (FDA) and fluorescein isothiocyanate (FITC) [5]-[7] have been used. Dinh et al. [8] combined the use of fluorescent stain PKH26 and microscopy rhizosphere chambers (micro-ROC), to study the development of the cyst nematode Heterodera schachtii, the root knot nematode Meloidogyne chitwoodi and the root lesion nematode Pratylenchus penetrans in roots of Arabidopsis thaliana. PKH26 is a yellow orange fluorescent dye (excitation max. $551 \mathrm{~nm}$ and emission max. $567 \mathrm{~nm}$ ) with an aliphatic tail and integrates into the lipid regions of the cell membrane [9]. Because of its extremely stable fluorescence, PKH26 is useful when labeled cells are to be followed for extended periods as demonstrated by the four-week retention time of PKH26 in mammalian cells [10].

Here, we describe a further elaboration and application of these techniques, to compare the course of infection of G. pallida in potato roots versus the trap crop Solanum sisymbriifolium. We applied these methods for in situ observations of $G$. pallida J2 infection in the non-host $S$. sisymbriifolium compared to its host $S$. tuberosum. We used micro-ROC coupled with the fluorescent stain PKH26, and compared this with destructively sampled acid fuchsin staining.

\section{Materials and Methods}

\subsection{Globodera pallida Culture and Preparation of Nematode Inoculum}

G. pallida was propagated on the susceptible potato cultivar "Desiree" in the greenhouse at $18^{\circ} \mathrm{C}$. The identity of G. pallida was confirmed by morphological and molecular methods [11]. The plants were watered twice daily with $75 \mathrm{ml}$ of water and fertilized three times per week with 20-20-20 all-purpose fertilizer. After 16 weeks of growth, cysts were recovered from soil by the Fenwick can method [12] and stored at $4^{\circ} \mathrm{C}$.

To obtain second stage hatched juveniles, 20 cysts were placed in $2.54 \mathrm{~cm}^{2}$ nylon mesh bags (McMaster Carr, USA), and dipped in $0.5 \%$ bleach for $5 \mathrm{~min}$, rinsed thoroughly with sterile distilled water). After hydration in sterile distilled water for 3 days [13], hatching was induced by placing cysts in potato root diffusate containing gentamicin $(1.5 \mathrm{mg} / \mathrm{ml})$ and nystatin $(0.05 \mathrm{mg} / \mathrm{ml})$ at $20^{\circ} \mathrm{C}$ in sterile 6 -well plate. After 2 weeks the hatched juveniles were collected and incubated overnight in a solution containing $100 \mu \mathrm{g} / \mathrm{ml}$ each of ampicillin and streptomycin (w/v) on a rocker (Standard Analog Rocker, VWR, USA) at $22^{\circ} \mathrm{C}$. The nematode suspension was fur- 
ther treated with benzethonium chloride $(0.125 \%)$ by continuous shaking for $20 \mathrm{~min}$, washed 8 times by centrifugation at $4000 \mathrm{rpm}$ for $10 \mathrm{~min}$, and re-suspended in sterile distilled water [14]. Collected J2s (300 J2s/100 $\mu \mathrm{L}$ ) were suspended in a solution of PKH26 $\left(4 \times 10^{-6} \mathrm{M}\right.$, as per manufacturer's protocol, Sigma Aldrich, USA), incubated for 10 mins at $22^{\circ} \mathrm{C}$ with intermittent shaking ( 3 times for $15 \mathrm{~s}$ ), and washed 5 times in sterile distilled water. The stained J2s were transferred to $0.01 \%$ sterile agarose [15]. Plantlets were inoculated with $100 \mu \mathrm{L}$ solution containing $2.5 \mathrm{~J} 2 \mathrm{~s} / \mu \mathrm{L}$ [8]. Inoculated plants were maintained under greenhouse conditions at $18^{\circ} \mathrm{C} \pm 2^{\circ} \mathrm{C}$, 16 hours light and 8 hours dark cycle for 20 days. Six replicates of each plant species were observed every two days for 20 days under an inverted microscope (Leica, DMI 3000B, Germany). Fluorescing nematodes were detected with a rhodamine filter.

\subsection{Plant Material and Micro-Rhizosphere Chambers}

Potato plants (Solanum tuberosum cv. "Desiree”) were grown in standard tissue culture media [16]. Solanum sisymbriifolium seeds were planted in plastic seed trays. One-week-old tissue culture potato plantlets and twoweek-old S. sisymbriifolium seedlings were transplanted into micro-ROC chambers (Advance Science Tools LLC, WA) and kept in the greenhouse at $18^{\circ} \mathrm{C} \pm 2^{\circ} \mathrm{C}$ for 3 weeks for development of roots. Sunshine mix (Sun Gro Horticulture, MA, USA) was used for the growth medium. Plants were watered once daily with $25 \mathrm{ml}$ of water and fertilized with water soluble 20-20-20 all-purpose fertilizer, three times weekly.

To determine the efficacy of staining with PKH26, a second set of $S$. tuberosum and S. sisymbriifolium grown in micro-ROC were inoculated with a similar method as above, but development of G. pallida was observed by using the standard acid fuchsin method [17]. Three replicates of S. tuberosum and S. sisymbriifolium were destructively sampled every two days up to 20 days post infection. Roots were stained with acid fuchsin [17] and observed under a stereo-microscope (Leica Microsystems, Germany). The development of G. pallida at each life stage was recorded.

\subsection{Statistical Analysis}

Counts of the different development stages were subjected to analysis of variance using generalized linear mixed effects procedure of $S A S^{\circledR}$ software Proc Glimmix [18]. The plant species, days post infection (2, 4, 6 and 8 days), and type of stain used were treated as fixed effects in the model. Main (individual effect of each treatment factor) and interaction effect (two way interactions of individual treatment factors) among fixed factors were tested. Data being unbalanced (due to three replicates for acid fuchsin and 6 replicates for PKH26), the Kenward-Roger approximation was used to adjust the denominator degrees of freedom, and the estimated standard errors for main and interaction effects [19]. Level of significance was set for $P \leq 0.05$. Post-hoc multiple pairwise comparisons of treatment group means were done with the Tukey-Kramer adjustment (Tukey's Honest Significant Difference test) to control for the Type I error rate. The packages, "ggplot2" [20] in the freeware R 3.2 [21] was used to plot the graphs. For cross-comparison of the results from the linear mixed effect model fitted to combined data, an independent standard ANOVA model on J2 counts from plant samples stained with acid fuchsin, which ignored the random effects, and a separate linear mixed effect model on J2 counts from plant samples stained with PKH26 only were also performed. The plants species and days post infection (dpi) were the only fixed effects in these models. Data analyzed spanned across 10 time points $(2,4,6,8,10,12,14$, 16, 18 and 20 dpi) for model fitted to samples stained with acid fuchsin only. Data for J2 counts are reported.

\section{Results}

\subsection{Comparison of PKH26 versus Acid Fuchsin}

Application of the fluorescent stain PKH26 coupled with micro-rhizosphere chambers allowed for in situ observations of G. pallida J2 infection in the non-host S. sisymbriifolium compared to its host S. tuberosum. Immediately after labelling with PKH26, the entire bodies of G. pallida J2s were clearly visible with fluorescence microscopy (Figure 1) and remained visible for the 20-day duration of the study. Labelled J2s successfully located and penetrated roots of both S. tuberosum and S. sisymbriifolium. No significant difference $(P \geq 0.90)$ in G. pallida numbers were found whether stained with PKH26 or acid fuchsin for either plant species indicating no toxic effect from the vital stain (Figure 2). Overall, more nematodes were observed in S. tuberosum than in S. sisymbriifolium roots. 


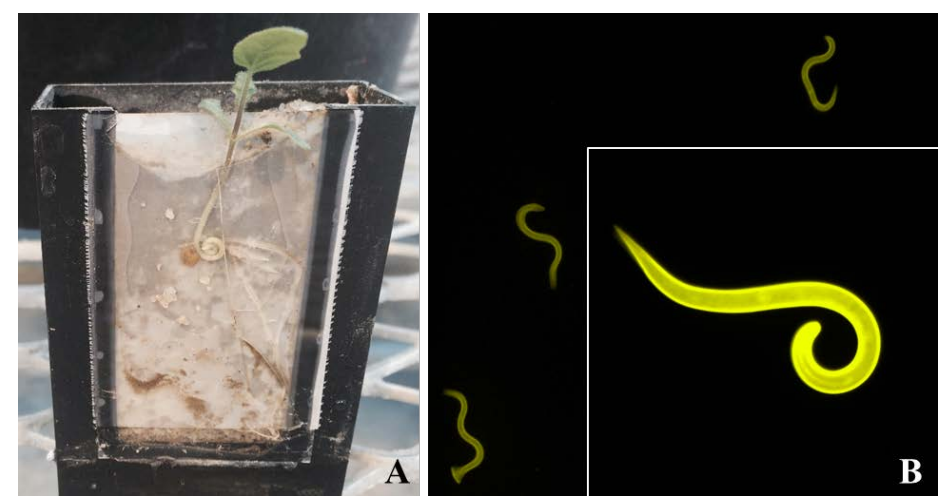

Figure 1. Solanum sisymbriifolium (A) growing in microscopy rhizosphere chambers (micro-ROC); PKH26 stained G. pallida J2s before inoculation (B).

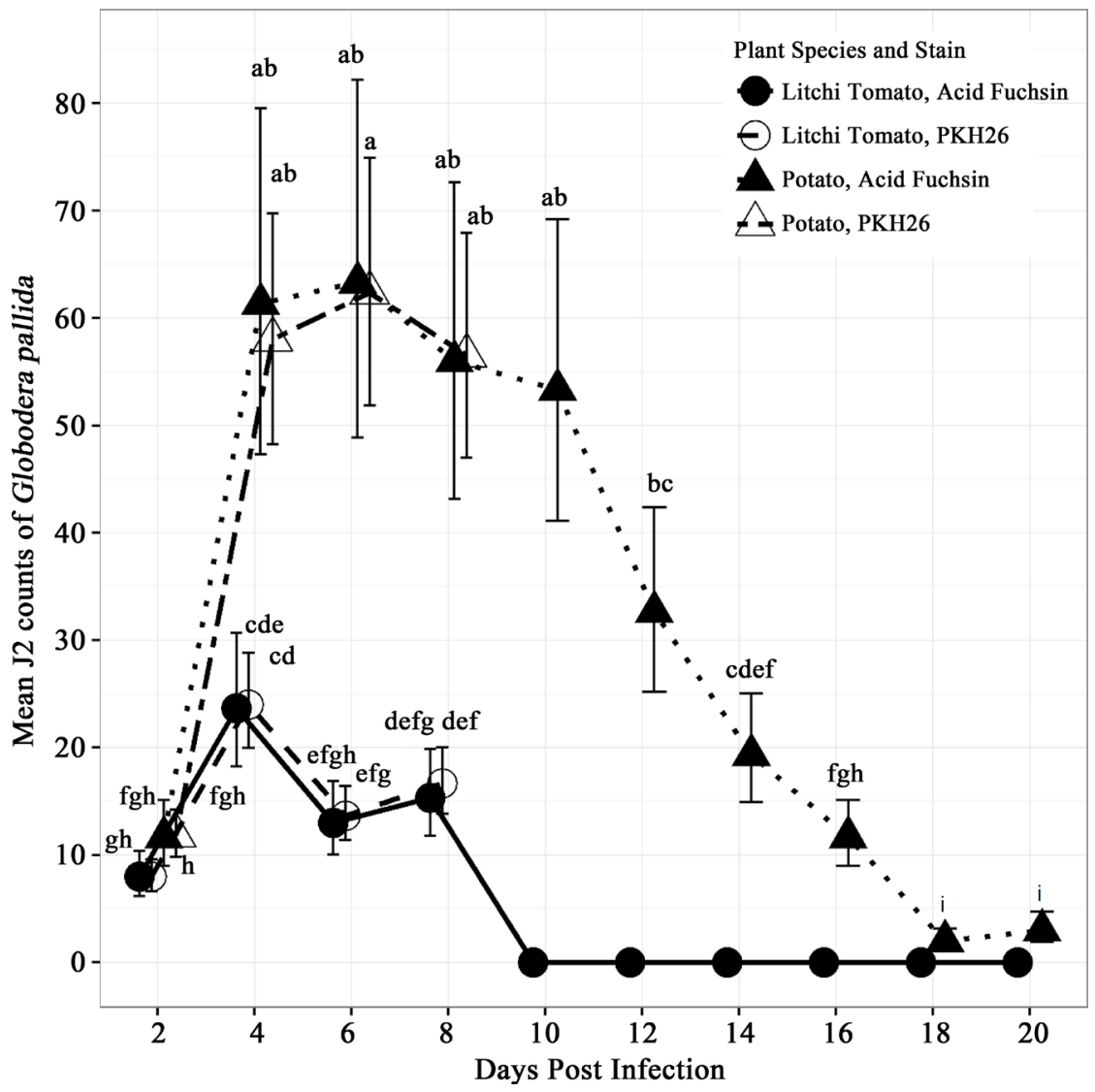

Figure 2. Mean J2 counts ( $\pm 95 \%$ confidence interval) of Globodera pallida at different dpi (days post infection) in acid fuchsin and PKH26 stained roots of Solanum tuberosum and Solanum sisymbriifolium grown in micro-ROC. Data points with different letters are significantly different.

PKH26 combined with micro-ROC allowed in situ observation of G. pallida in its host S. tuberosum including J2 migration through root tissue (Figure 3), and molt to J3 (Figure 3). Female development was not observed with PKH26 stained nematodes, but was observed when stained with acid fuchsin.

\subsection{G. pallida Development in S. sisymbriffolium Compared to Potato}

At 2 DPI, PKH26 stained G. pallida were clearly observed migrating intercellularly through root tissues of both S. tuberosum and the non-host S. sisymbriifolium (Figure 3). The numbers of J2s in roots increased from day 2 
through day 8 and subsequently declined, for both plant species (Figure 2). At 2 DPI, the numbers of J2s penetrating and migrating in S. sisymbriifolium was the same for S. tuberosum. However, at 4 DPI, presence of J2 in S. sisymbriifolium roots was significantly less than in S. tuberosum. The maximum number of J2s in S. tuberosum at 6 days (mean \pm SE: $63 \pm 3$ ) was nearly three times higher $(P<0.0001)$ than the maximum observed in $S$. sisymbriifolium at 4 days (24 \pm 2 ) (Figure 2).

No live J2s were observed in S. sisymbriifolium roots stained with either acid fuchsin or PKH26 after 8 days (Figure 3). At 10 DPI and after, dead and deformed PKH26 labelled nematodes were still visible in roots of $S$. sisymbriifolium, but not in the acid fuchsin stained roots (Figure 3 and Figure 4).

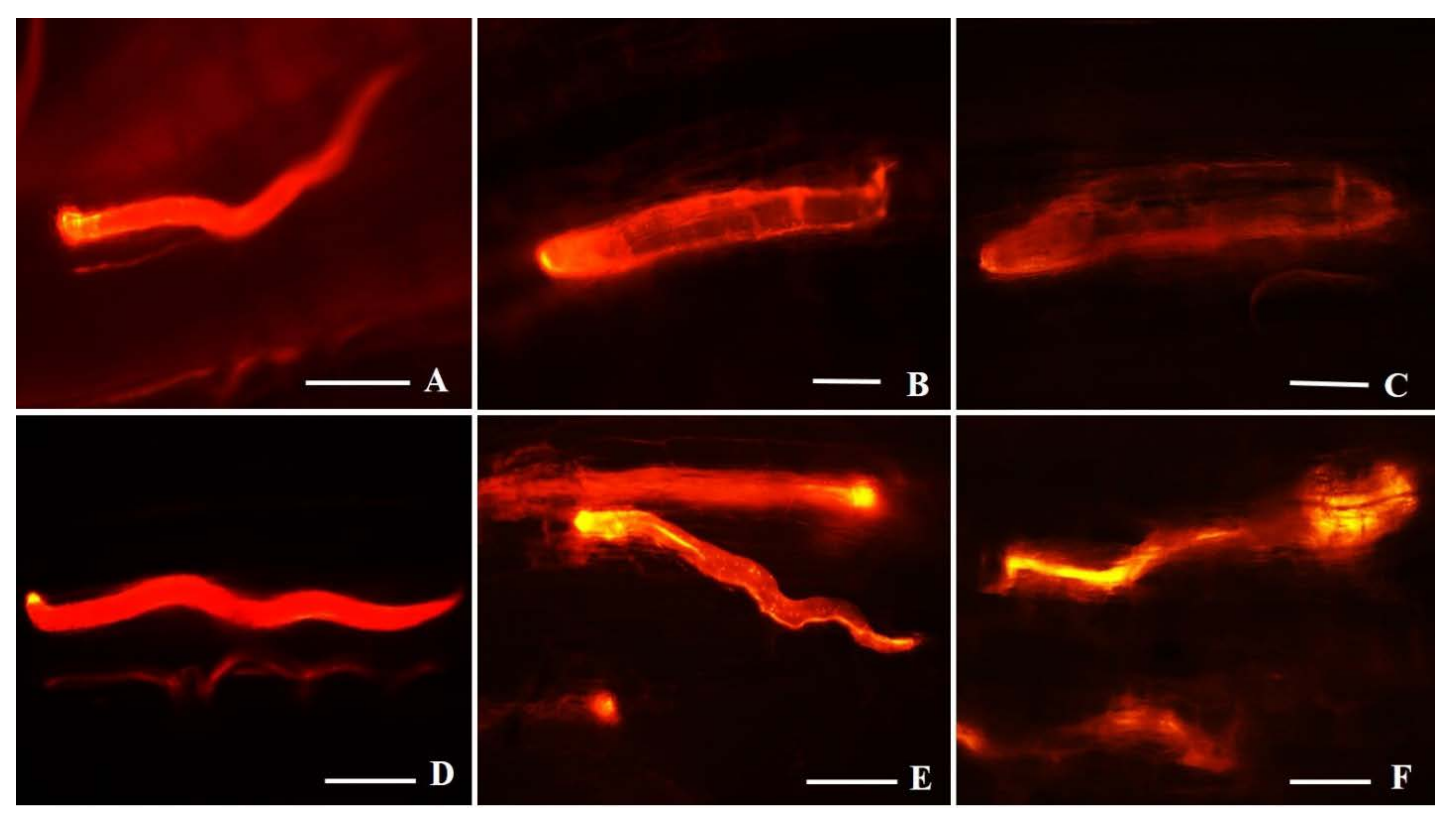

Figure 3. PKH26 stained Globodera pallida in the roots of Solanum tuberosum ((A) - (C)) and Solanum sisymbriifolium ((D) - (F)) up to 20 dpi (days post infection). (A) and (D): 2 dpi; (B) and (E): 10 dpi; (C) and (F): 20 dpi. Dead and deformed nematodes can be seen in S. sisymbriifolium roots in (E) and (F), scale bar $=50 \mu \mathrm{M}$.
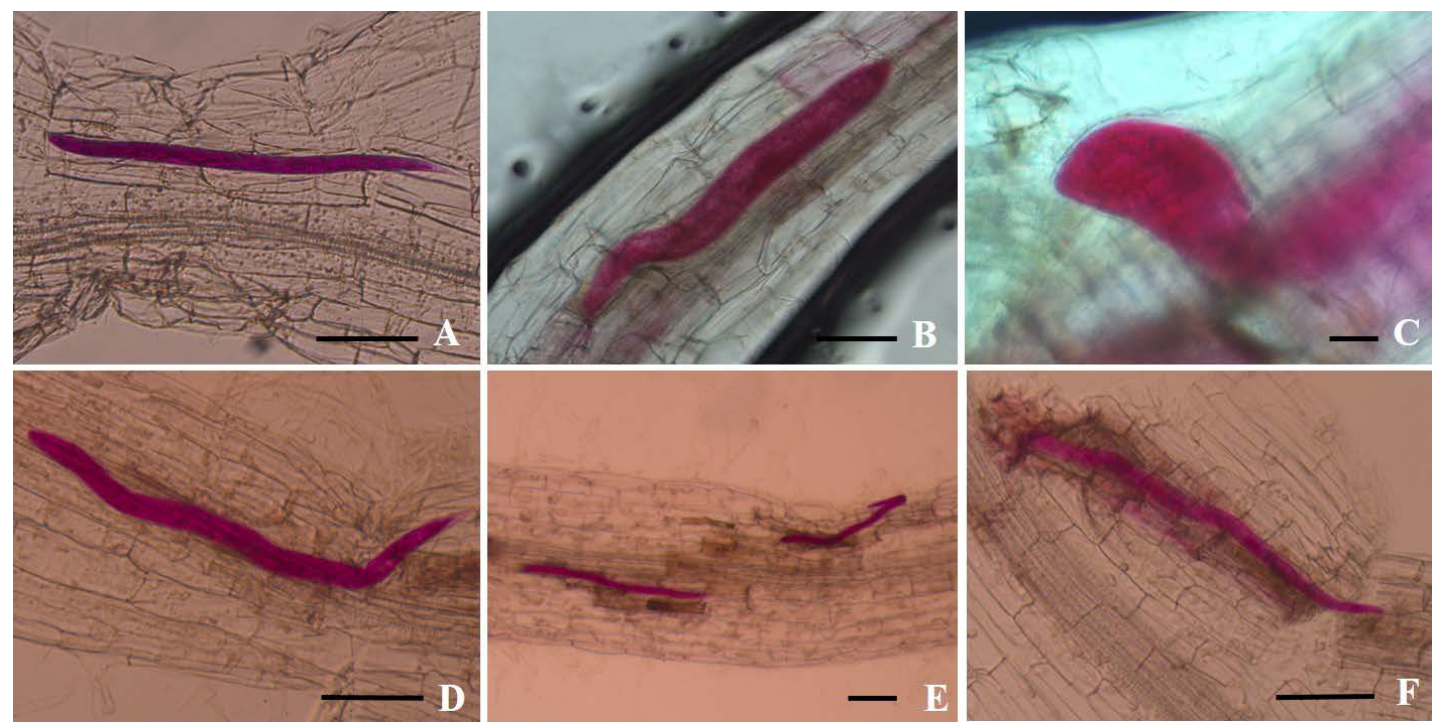

Figure 4. Acid fuchsin stained roots of Solanum tuberosum ((A): 2 dpi, (B): 12 dpi, (C): 20 dpi) and Solanum sisymbriifolium ((D): 2 dpi (days post infection), (E): 4 dpi, (F): 8 dpi after Globodera pallida inoculation. J3s (B) and J4s (C) were observed in S. tuberosum roots. In S. sisymbriifolium, localized cell death was observed at 4 dpi (E) and 8 dpi (F); scale bar $=50 \mu \mathrm{M}$. 
In S. tuberosum, G. pallida continued to develop into third and fourth stage juveniles within 16 dpi (Figure 4). Whereas in S. sisymbriifolium, G. pallida died within the root tissue, and no further development of the nematode occurred (Figure 3 and Figure 4). Resistance in S. sisymbriifolium was expressed rapidly, and plant cell death was observed at 4 DPI (Figure 5). Solanum tuberosum infected cells did not show any morphological changes as observed under epifluorescence (Figure 5(B)) but S. sisymbriifolium infected root cells auto-fluoresced, and the nematode was dead (Figure 5(C) and Figure 5(D)). Similarly, invaded cells of S. sisymbriifolium from acid-fuchsin-stained samples, had thickened walls and were discolored (Figure 4), while this response was not observed in S. tuberosum.

\section{Discussion}

Staining with PKH26 or acid fuchsin gave virtually identical results for both plant species. Thus, use of the vital stain PKH26 apparently had no deleterious effect on either infectivity or detection of G. pallida J2s. Also, these results suggest that the stain remains incorporated in the nematode body through subsequent molts. A promising research direction will be the use of this stain to follow nematode movement and development within the root. Understanding the time course of infection and progress of resistance in the non-host S. sisymbriifolium in comparison to its host $S$. tuberosum will guide subsequent assays to determine the genetic response to infection.

We showed that $G$. pallida was able to penetrate the roots of both plant species, but, the number of nematodes entered was much reduced in S. sisymbriifolium as compared to $S$. tuberosum. Dead nematodes were found in the roots of S. sisymbriifolium at $4 \mathrm{dpi}$, which shows total inhibition of G. pallida development in S. sisymbriifolium as compared to susceptible potato. Our microscopic observations indicate that cell necrosis develops around the nematode which may indicate that $S$. sisymbriifolium establishes a hypersensitive response when invaded by G. pallida. Anwar and McKenry [22] used host cell necrosis as a measure of hypersensitive response in resistant Vitis spp. infected with Meloidogyne arenaria resulted in halted or delayed migration of nematodes in resistant plant roots. Inhibition in the development of G. pallida in S. sisymbriifolium after penetration was
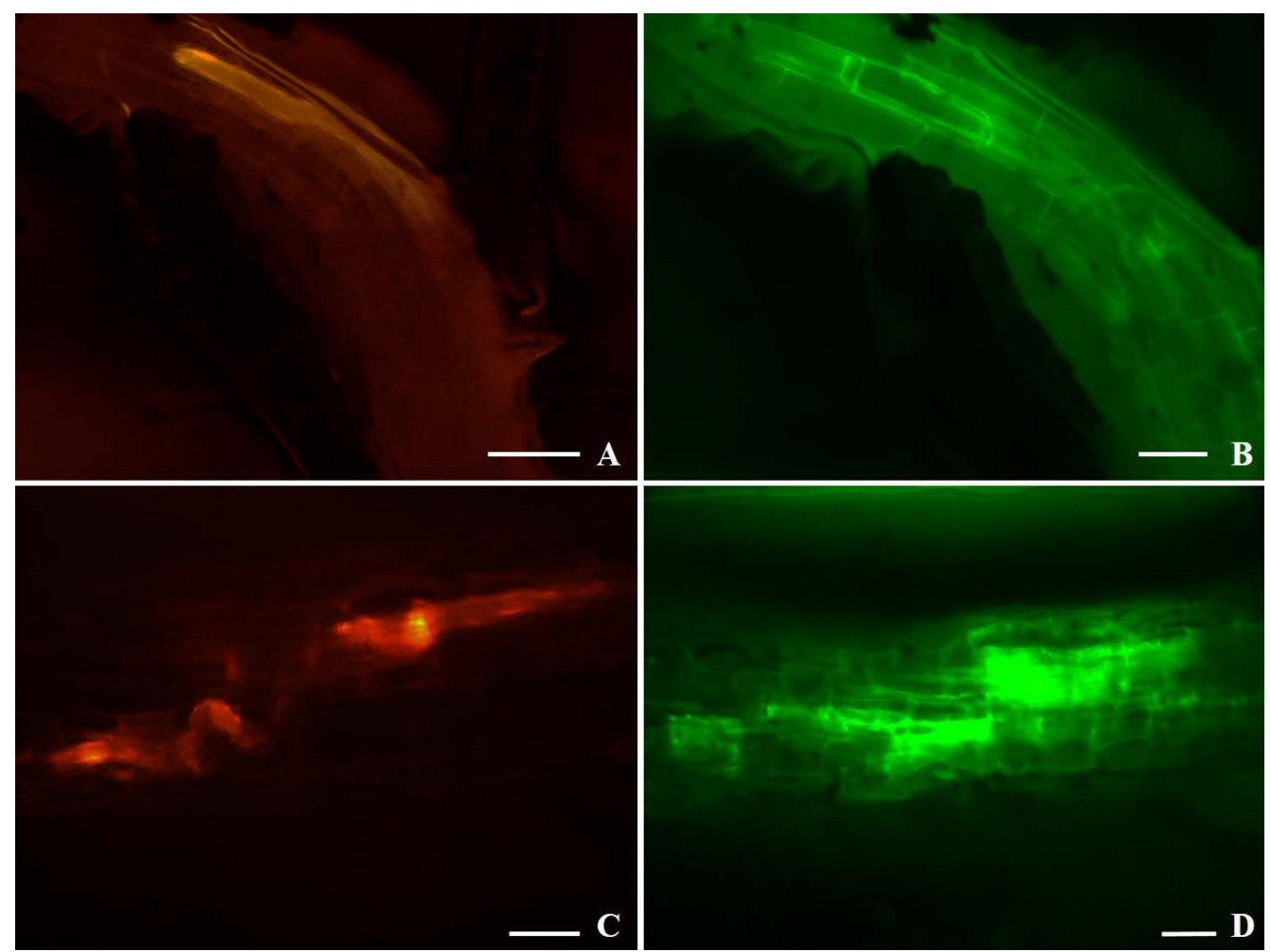

Figure 5. (A) and (C) show PKH26 stained G. pallida in Solanum tuberosum and Solanum sisymbriifolium, respectively; (B) and (D) show the infected cells of $S$. tuberosum and S. sisymbriifolium, respectively under green fluorescence. Compared to S. tuberosum root cells (B) G. pallida infected S. sisymbriifolium root cells exhibited cell-wall thickening and death (D); scale bar $=50 \mu \mathrm{M}$. 
also reported by Sasaki-Crawley et al. [2] [23], however, contradictory to our findings, they reported the presence of G. pallida in S. sisymbriifolium up to 5 weeks post infection.

\section{Conclusion}

Micro-ROC coupled with PKH26 was first used by Dinh et al. [8] for the live in planta observation of Pratylenchus penetrans, Heterodera schachtii and Meloidogyne chitwoodi in the roots of Arabidopsis thaliana. Our study demonstrates the efficacy of live fluorescent staining with PKH26 method to study the course of development and interaction of a cyst nematode in a susceptible and resistant plant species. PKH26 staining did not affect the infection process and was an effective tool for nematode developmental studies. The advantages of using PKH26 coupled with micro ROC to study dynamics of G. pallida allowed the visualization of dead and deformed J2s in the infected host plant cells, and to better understand the timing of the plant response. Autofluoresence of cells infected with G. pallida was observed in S. sisymbriifolium roots but not in S. tuberosum roots. Infected S. sisymbriifolium cells had thickened walls and were dark in color indicative of localized cell death. Necrosis and degeneration of syncytium have been observed in resistant soybean cultivar as early as 48 hours or after 8 - 10 dpi with Heterodera glycines, depending on the source of resistance [24] [25]. Localized gene expression occurring at the feeding site during its establishment can lead either to the formation of a nurse cell called a syncytium as observed in a susceptible reaction [26], or to a localized cell death resulting in death of the nematode as observed in resistant or immune responses [27].

\section{Acknowledgements}

This research was supported by funding from the Idaho Potato Commission, the Northwest Potato Research Consortium and by USDA-APHIS Farm Bill 10007.

\section{References}

[1] Scholte, K. (2000) Screening of Non-Tuber Bearing Solanceae for Resistance to and Induction of Juvenile Hatch of Potato Cyst Nematodes and their Potential for Trap Cropping. Annals of Applied Biology, 136, 239-246. http://dx.doi.org/10.1111/j.1744-7348.2000.tb00030.x

[2] Sasaki-Crawley, A., Curtis, R., Birkett, M., Powers, S., Papadopoulos, A., Pickett, J., Blackshaw, R. and Kerry, B. (2010) Signaling and Behavior of Potato Cyst Nematode in the Rhizosphere of the Trap Crop, Solanum sisymbriifolium. Aspects of Applied Biology, 103, 45-51.

[3] Ouden, H.D. (1958) A New Method for Culturing Plants Enabling the Observation of Nematodes on Growing Roots. T. Pl.ziekten, 64, 269-272.

[4] Atkinson, H.J., Urwin, P.E., Clarke, M.C. and McPherson, M.J. (1996) Image Analysis of the Growth of Globodera pallida and Meloidogyne incognita on Transgenic Tomato Roots Expressing Cystatins. Journal of Nematology, 28, 209-215.

[5] Bird, A.F. (1979) A Method of Distinguishing between Living and Dead Nematodes by Enzymatically Induced Fluorescence. Journal of Nematology, 11, 103-105.

[6] Schroeder, N.E. and MacGuidwin, A.E. (2007) Incorporation of a Fluorescent Compound by Live Heterodera glycines. Journal of Nematology, 39, 43-49.

[7] Goto, D.B., Fosu-Nyarko, J., Sakuma, F., Sadler, J., Flottman-Reid, M., Uehara, T., Kondo, N., Yamaguchi, J. and Jones, G.K. (2010) In Planta Observation of Live Fluorescent Plant Endoparasitic Nematodes During Early Stages of Infection. Nematological Research, 40, 15-19. http://dx.doi.org/10.3725/jin.40.15

[8] Dinh, P.T.Y., Knoblauch, M. and Elling, A.A. (2014) Nondestructive Imaging of Plant-Parasitic Nematode Development and Host Response to Nematode Pathogenesis. Phytopathology, 104, 497-506. http://dx.doi.org/10.1094/PHYTO-08-13-0240-R

[9] Wallace, P.K., Tario, J.D.Jr., Fisher, J.L., Wallace, S.S., Ernstoff, M.S. and Muirhead, K.A. (2008) Tracking Antigen-Driven Responses by Flow Cytometry: Monitoring Proliferation by Dye Dilution. Cytometry, 73A, 1019-1034. http://dx.doi.org/10.1002/cyto.a.20619

[10] Herrmann, I., Baeuerle, P.A., Friedrich, M., Murr, A., Filusch, S., Ruttinger, D., Majdoub, M.W., Sharma, S., Kufer, P., Raum, T. and Munz, M. (2010) Highly Efficient Elimination of Colorectal Tumor Initiating Cells by an Epcam/cd3Bispecific Antibody Engaging Human T Cells. PLoS ONE, 5, e13474. http://dx.doi.org/10.1371/journal.pone.0013474

[11] Skantar, A.M., Handoo, Z.A., Carta, L.K. and Chitwood, D.J. (2007) Morphological and Molecular Identification of 
Globodera pallida Associated with Potato in Idaho. Journal of Nematology, 39, 133-144.

[12] Fenwick, D.W. (1940) Methods for the Recovery and Counting of Cysts of Heterodera schachtii from Soil. Journal of Helminthology, 18, 155-172. http://dx.doi.org/10.1017/S0022149X00031485

[13] Palomares-Ruis, J.E., Jones, J.T., Cock, P.J., Castillo, P. and Blok, V.C. (2013) Activation of Hatching in Diapaused and Quiescent Globodera pallida. Parasitology, 140, 445-454. http://dx.doi.org/10.1017/S0031182012001874

[14] Upadhyay, D., Kooliyottil, R., Mandjiny, S., Inman III, F.L. and Holmes, L.D. (2013) Mass Production of the Beneficial Nematode Steinernema carpocapsae Utilizing a Fed-Batch Culturing Process. eSci Journal of Plant Pathology, 2, 52-58.

[15] Sobczak, M., Avrova, A., Jupowicz, J., Phillips, M.S., Ernst, K. and Kumar, A. (2005) Characterization of Susceptibility and Resistance Responses to Potato Cyst Nematode (Globodera spp.) Infection of Tomato Lines in the Absence and Presence of the Broad-Spectrum Nematode Resistance Hero Gene. Molecular Plant Microbe Interactions, 18, 158-168. http://dx.doi.org/10.1094/MPMI-18-0158

[16] Murashige, T. and Skoog, F. (1962) A Revised Medium for Rapid Growth and Bio Assays with Tobacco Tissue Cultures. Physiologia Plantarum, 15, 473-497. http://dx.doi.org/10.1111/j.1399-3054.1962.tb08052.x

[17] Byrd Jr., D.W., Kirkpatrick, T. and Barker, K.R. (1983) An Improved Technique for Clearing and Staining Plant Tissue for Detection of Nematodes. Journal of Nematology, 14, 142-143.

[18] SAS Institute (2011) The SAS System for Windows. Release 9.2., SAS Institute Inc., Cary, NC.

[19] Littell, R.C., Milliken, G.A., Stroup, W.W., Wolfinger, R.D. and Schabenberger, O. (2006) SAS for Mixed Models, Second Edition. SAS Institute Inc., Cary, NC.

[20] Wickham, H. (2009) Ggplot2: Elegant Graphics for Data Analysis. Springer, New York.

[21] R Core Team (2015) R: A Language and Environment for Statistical Computing. R Foundation for Statistical Computing, Vienna, Austria. http://www.R-project.org/

[22] Anwar, S.A. and McKenry, M.V. (2000) Penetration, Development and Reproduction of Meloidogyne arenaria on Two New Resistant Vitis spp. Nematropica, 30, 9-17.

[23] Sasaki-Crawley, A., Curtis, R., Birkett, M., Papadopoulos, A., Blackshaw, R. and Pickett, J. (2012) The Use of Pluronic F-127 to Study the Development of the Potato Cyst Nematode, Globodera pallida. Nematology, 14, 869-873. http://dx.doi.org/10.1163/156854112X631944

[24] Acedo, J.R., Dropkin, V.H. and Luedders, V.D (1984) Nematode Population Attrition and Histopathology of Heterodera glycine-Soybean Associations. Journal of Nematology, 16, 48-56.

[25] Mahalingam, R. and Skorupska, H.T. (1996) Cytological Expression of Early Response to Infection by Heterodera glycines Ichinohe in Resistant PI 437654 Soybean. Genome, 39, 986-998. http://dx.doi.org/10.1139/g96-123

[26] Rehman, S., Postma, W., Tytgat, T., Prins, P., Qin, L., Overmars, H., Vossen, J., Spiridon, L.N., Petrescu, A.J., Goverse, A., Bakker, J. and Smart, G. (2009) A Secreted SPRY Domain-containing Protein (SPRYSEC) from the Plantparasitic Nematode Globodera rostochiensis Interacts With a CC-NB-LRR Protein from a Susceptible Tomato. Molecular Plant-Microbe Interactions, 22, 330-340. http://dx.doi.org/10.1094/MPMI-22-3-0330

[27] Moloney, C., Griffin, D., Jones, P.W., Bryan, G.J., McLean, K., Bradshaw, J.E. and Milbourne, D. (2010) Development of Diagnostic Markers for use in Breeding Potatoes Resistant to Globodera pallida Pathotype Pa2/3 Using Germplasm Derived from Solanum tuberosum ssp. Andigena CPC 2802. Theoretical and Applied Genetics, 120, 679-689. http://dx.doi.org/10.1007/s00122-009-1185-0 